\title{
The Treatment Technology of Recycling Scrap Wire and Cable
}

\author{
Sa Xiao ${ }^{1,}$ a , Wei Xiong ${ }^{1, b}$, Lijun Wang ${ }^{2, \mathrm{c}}$, Qiaolin Ren ${ }^{2, \mathrm{~d}}$ \\ ${ }^{1}$ College of Electrical Engineering and New Energy, China Three Gorges University, Yichang, \\ Hubei, 443000, China \\ ${ }^{2}$ Maintenance company of Xiaogan Power Supply Company, Xiaogan, Hubei, 443000,China \\ a1028628389@qq.com, b80850576@qq.com, c1033287008@qq.com, d1634436353@qq.com
}

\begin{abstract}
Keywords: Scrap wire and cable, Stripping machine, Mechanical crushing and classification, High pressure water jet, Chemical treatment, Freezing, Heat recovery, Ultrasonic separation;

Abstract. With the development of modern industry and communications industry, a variety of metal wires are used more and more. At the same time, the number of cables required waste recycling is also increasing and the environment pollution has become more serious. Currently, cable for waste recycling technology is mainly as follows: stripping machine technology, mechanical crushing sorting technology, high-pressure water jet recovery technology, chemical processing technology, cryogenic technology, heat recovery technology and ultrasonic separation technology. In this paper, It conducted a comprehensive analysis of various techniques, and conduct research status.
\end{abstract}

\section{Introduction}

Copper is an important raw material of the national economy, but that relative lack of copper resources in our country. The current annual demand of 1.1 million tons meter, our confession is only 650,000 tons of copper resources, the number of imported more than 400,000 tons. Which imports of copper resources in more than half of that is based on secondary sources of copper in the form of scrap copper appears. While imports of scrap copper, scrap wire and cable majority again, in more than 20 tons. Imports of scrap copper increased year by year may be caused by the tariff policy tilt, because the waste copper smelting energy consumption is only $1 / 8$ of the copper ore, or even can be directly processed into finished products.

Faced with this reality, people have to look for new and more effective way to solve the problem of waste recycling wire and cable to industrialization. Some industrial countries and regions in this regard has made a successful exploration, wire and cable processing domestic waste entered into a large-scale, automated, non-polluting stage, but in the country has not been universal. In this paper, a variety of methods that current situation will to be study to solve the domestic waste wire processing issues raised most appropriate solution.

\section{Waste treatment methods Power Cable}

In advance of the scrap metal recycling metal pretreatment is a very important step in secondary use, process and equipment used in metal and mineral similar [1]. Currently, cable for waste recycling technology is mainly as follows: stripping machine technology, mechanical crushing sorting technology, high-pressure water jet recovery technology, chemical processing technology, cryogenic technology, heat recovery technology and ultrasonic separation technology.

Stripping Machine Technology .Stripping machine technology have a large number applications in large wire. Its working principle is relatively simple, that mainly driven by a stepping motor and wheel clamped wire movement, and in order to achieve cutting and peeling, the specific process is as follows: Wire and cable through the right wheel drive stepper motors move around, stepper motor control through the cutter, cutter relative displacement, enabling the peeling and cutting lines. At the same time, not only through the left wheel drive motor is sandwiched move around wires and cables; the twisted wire wheel stepping motor driven, lower roller back and forth in the opposite direction, to achieve twisting. 
Mechanical crushing sorting technology .Mechanical crushing separation technology based on the principle of different functions can be divided into the following two units: crushing and sorting. Scrap cable is first broken down into a certain scale unit particle unit, and then get the pure copper and plastic through sorting unit role. Currently the most used is the mechanical crushing cell technology, which is a direct reference mature technology Mining industry sectors, including extrusion, friction, shear, impact, splitting, bending, etc. to complete the process. Equipment used mostly by simple transformation from mineral device, not specifically designed for this device. In contrast, the sorting unit Optional numerous methods currently used more there are sorting, screening, choose water, electricity choice. The specific choice of selecting the appropriate sorting method according to the requirements of the separation rate.

High pressure water jet recycling technology .High pressure water jet work principle is very simple, and the process is not complicated, that mainly working principle is cut uniform movement of scrap cable by cold. Due to the high precision split only split the cable outer plastic, it does not produce the thermal phenomena in the work process, as the same time there is not heat impact on the quality of the metal will not bring any change.

HE Kai [2], etc. for this technology are constantly in-depth study and eventually to cut through experiments using scrap cable engineering projects. In the present invention, the pressure of the water jets have certain restrictions, that must be higher than $55 \mathrm{MPa}$, as the same time, the mechanism cutting parameters of device should be set accurately, and waste cable wound on a reel needs, so as to be more convenient and accurate cutting.

Chemical treatment technology .Chemical treatment is the use of copper or aluminum metal stability that does not react chemically with some solutions, but these can be a good solution and dissolve the outer layer of wire and cable insulation. After the chemical reaction, it is the only metal, just to the metal filter can achieve their goals.

Park Chang-ji [3] etc. mainly on the extraction of metal wire and wire copper made a thorough study, and achieved certain results. Heating using warm water or steam, scrap wire reclaimed oil mixed together into a furnace, by melting scrap cable insulation sheath, filtered, then recovered by evaporation of recycled oil. Then use physical techniques for metal line filtration, distillation oil removal treatment can eventually be recycled. Bai yunhe[4] invention is the use of metal catalyzed decomposition outer cable insulation to achieve the purpose of separation insulating sheath and metal.

Cryogenic processing technology .The basic principle is the use of cryogenic refrigerant to change material and enable the material become brittle so that pulverized.

Zhu Ling, Cui Hong-xiang[5,6] etc. design a waste wires and cables use ammonia stripping method according to the principles described above. The experimental results show high recovery efficiency of this method, and the entire process in addition to less human intervention, a high degree of automation. During the experiment, in order to protect job security and personal safety, that avoid using those toxic, low melting point and is prone to explosive refrigerant. At this stage, the most used is ammonia, CO2, the second is used more there Freon R12, freon and other refrigerants R14.

Heat Recycling Technology .Thermal decomposition technology is currently the most use a cable recycling of waste treatment technology. Which the content of oxygen requirements are very low, almost to a vacuum state, the process is carried out in a closed container environment, and in a certain pressure on the thermal decomposition for the insulating sheath, the final product of the oil and gas, can be reused after the conversion of oil and gas. Basfar A A[7] in-depth study for a class of materials PVC cable through experiments Guo Yulong[8] in-depth study of the computer cable. Chen Zhongzhu[9] synthesis two different methods, there are heating technology and mechanical separation technology. Wasting wire placed insulating oil separation at high temperature, the use of impact action separate drive means to achieve complete separation of the sheath and the metal purposes.

Ultrasonic separation and recovery technology .Ultrasonic separate transmission medium is water, resulting in cavitation energy through its transmission, this phenomenon will have a dramatic 
vibration, vibration force is sufficient separation insulation and metal, good effect, has been widely recognized. Tried and tested, as in the work process does not require multiple wire and cable cutting, thus greatly reducing the losses and consumption of copper.

Fan Naifeng[10] etc. investigated water temperature, the length of the wire was cut and liquid-solid ratio influence on the separation efficiency. Tests show that the use of such technology can reduce the loss of copper, and can increase the effective recovery of reusable resources. Due to thermal expansion and contraction as well as joint action of ultrasonic cavitation, plus external thermostat $60^{\circ} \mathrm{C}$ conditions, the three combine to achieve maximum separation rate. And the cable length and separation rate is also a great relationship, the longer the length of the cable, the longer the time of the separation process, the lower the separation rate, on the contrary, the shorter the separation process, the higher the separation rate, which is particularly for those single and multi-core effect is most obvious. In addition, the share of the mass ratio of copper have a great influence on the separation, the higher the proportion that is relatively the less insulation, the smaller copper wire binding, which separation of the insulation and copper play to good role in promoting. From the whole, solid-liquid ratio higher separation rate is lower, but the local fluctuation.

Liu Yong[11] studying Ultrasonic separation which steps are relatively simple, but requires the cable crushed, which is cut into pellets to length ratio of the metal conductor and cable diameters between particles remain within a fixed range, general 1: $05 \sim 5$, the water temperature should be below the boiling point of the medium. Meanwhile ultrasonic temperature, ultrasonic frequency, ultrasonic energy density and treatment time has a certain range.

\section{Conclusions}

Based on the above analysis of the advantages and disadvantages of several methods to dispose of used wires and cables, according to China's national conditions, the recovery of waste wire processing needs further research and exploration to further reduce copper consumption, while increasing production while also focusing on environmental protection, reduce emissions, improve energy efficiency secondary. There should be based on the reality of the situation and the level of economic development and industry trends choose recycling method to improve the existing technology continues to improve and innovate, but also should learn foreign advanced technology and widely applied to the final of scrap wire and cable treatment projects in the past, for China's wire and cable treatment and recovery of waste to contribute.

\section{Acknowledgements}

This work was financially supported by the Electric vehicle development trends and their impact on the distribution network research projects (SDHZ2015195), Pyrolysis waste cable research projects (SDHZ2015258) and Electric vehicle charging and distributed solar consumptive joint optimization of research projects (2015KDW10). The first two items from the Hubei Electric Power Company Xiaogan Power Supply Company research, the last one from the Provincial Key Laboratory of funds.

\section{Reference :}

[1] Hong Peiji. Introduce some foreign scrap copper recycling process [J]. China Resources Comprehensive Utilization, 1995:(07):30-33.

[2] He Kai, Li Jiuhua, Wei Shuguo. Based on the high-pressure water jet scrap wire cable recycling method and apparatus. China, 201310102732.7[P].2013-07-10.

[3] Piao Changji, Jin Dazhong. The use of renewable oils and oils extracted from scrap metal wire cable or a method and apparatus for waste tires: China, 200810210049.4[P].2008-12-31. 
[4] Bai Yunhe, Fan Hongbo. Wire and cable scrap metal recovery systems. China , 201210412167.X[P].2013-03-06.

[5] Zhu lin. A method of separation of scrap metal conductor wire and cable and plastic: China, 00111327.5[P].2002-03-20.

[6] Cui hongxiang, Wang Zhiyuan, Wan Jun. A use of liquid nitrogen cryogenic technology of waste plastic wire jacket stripping method and apparatus: China, 200810152904.0[P].2009-04-08.

[7] Basfar A A. Wire and cable materials research coupled PVC insulated flame retardant used [J]. Polymer degradation and stability, 2002,77(2):221-226.

[8] Guo Yulong, Wang Songtao, Liu Jingyang. Product analysis process waste electric Solutions [J]. Shenzhen University, 2009,26(3):246-249.

[9] Chen Zhongzhu. Waste wire separator: China, 200520086499.9[P].2007-4-25.

[10] Fan Naifeng, Luo Zhen, Li Yang. Waste cable recycling method is based on the principle of ultrasonic cavitation[J].2010,28 ( 6 ) : 63-66.

[11] Liu Yong, Zhang Shuting, He Yun. Stripped cable scrap metal and nonmetal method: China, 200810152868.8[P].2009-04-01. 